\title{
Control interno y gestión empresarial de centros comerciales peruanos en tiempos de la actual pandemia (2020)
}

\section{Manuel Alberto Espinoza Cruz, Ericka Nelly Espinoza Gamboa, Hugo Eladio Chumpitaz Caycho}

Facultad de Administración y Negocios Universidad Tecnológica del Perú

Facultad de Contabilidad y Finanzas Universidad Privada del Norte

Escuela de Posgrado Universidad César Vallejo

La presente investigación ha tenido por objetivo analizar en qué nivel se relaciona el control interno con la gestión empresarial de centros comerciales en tiempos de COVID-19. La búsqueda de eficacia y eficiencia óptima se hace muy necesaria dada la emergencia sanitaria, especialmente, cuando la pandemia y su tratamiento evolucionan y se adaptan. El trabajo se desarrolló en un distrito populoso de Lima, desde una dimensión financiera y operativa. Se pudo llegar a la conclusión de que, en el contexto de la actual pandemia, el control interno se relaciona significativamente con la gestión empresarial de centros comerciales.

Palabras clave: control interno, gestión empresarial, administración financiera, auditoría, COVID-19

\section{Controle interno e gestão empresarial de shopping centers peruanos, em tempos de atual pandemia (2020)}

O objetivo desta pesquisa foi analisar em que nível o controle interno está relacionado à gestáo empresarial de shopping centers, em tempos de COVID-19. A busca pela eficácia e eficiência ótimas é muito necessária, dada a emergência sanitária, especialmente quando a pandemia e seu tratamento evoluem e se adaptam. O trabalho foi desenvolvido em um bairro populoso de Lima, com dimensão financeira e operacional. Foi possível concluir que o controle interno está significativamente relacionado à gestão empresarial de shopping centers, em tempos de atual pandemia.

Palavras-chave: controle interno, gestão empresarial, gestão financeira, auditoria, COVID-19

\section{Internal control and business management of Peruvian shopping centers, in times of the current pande-} mic (2020)

The objective of this research has been to analyze at what level internal control is related to the business management of shopping centers, in times of COVID-19. The search for optimal effectiveness and efficiency is very necessary, given the health emergency, especially when the pandemic and its treatment evolve and adapt. The work was developed in a populous district of Lima, with a financial and operational dimension. It was possible to reach the conclusion that internal control is significantly related to the business management of shopping centers, in times of the current pandemic.

Keywords: internal control, business management, financial management, audit, COVID-19 


\section{Introducción}

El control interno es un apoyo instrumental para toda organización. Ya que la responsabilidad corresponde a todo componente humano de la institución, la gestión empresarial se convierte en el reflejo del monitoreo imperante. Los supervisores de todos los niveles deben ser respaldados por controles aceptables. Esta situación se trasluce, con mayor nitidez, cuando se acentúan debilidades en su manejo, como es el caso de los centros comerciales del distrito La Victoria de Lima, Perú. Tales situaciones se presentan por falta de cultura de control empresarial. Esto se evidencia, por ejemplo, en el caso reportado por Redacción El Popular (2020), que informó que determinados negociantes confeccionaban clandestinamente polos y ropa deportiva de marcas reconocidas internacionalmente en el centro comercial Sol de Fátima en el emporio comercial Gamarra del distrito La Victoria. En esta situación, hay un problema adicional de falta de seguridad ciudadana, colindante con el comercio informal, pues, antes de la pandemia del COVID-19, los negocios de confección clandestina eran del 70\%; luego de declararse la pandemia, subieron al $80 \%$. Con ello, el riesgo de los negocios aumentó (Yong, 2018).

En la tabla 1, se muestra el modelo de gestión empresarial que desde 2017 está vigente, tomando en cuenta el control interno. La tabla fue desarrollada sobre la base de lo establecido por el COSO ERM, que proviene del Comité de Organizaciones Patrocinadoras de la Comisión Treadway (en adelante, COSO) para la administración de riesgos empresariales (en adelante, ERM por sus siglas en inglés). Este enfoque enlaza el flujo del proceso ERM con los componentes del COSO.

Lo importante en los centros comerciales de Gamarra es el empuje de los negociantes, que conforman el emporio textil más grande del país. Esto explica que los riesgos sean mayores, más aún con el nivel de informalidad existente. Los controles apoyan a los gestores para que sus negocios sean más exitosos (Yong, 2018). Sin embargo, no se identifican como directamente responsables del control interno ni de los efectos negativos de un inadecuado manejo de los riesgos. En ese sentido, se necesita difundir el concepto de control interno (Gómez, 2001). Esta situación se hace ineludible ante las amenazas iniciales y las consecuencias en los negocios a raíz de la pandemia del COVID-19, que se ha expandido en todo el mundo y en países de habla hispana, como es el del presente caso (NaviaNúñez \& Stefanu-Lidorikiotu, 2020).

\section{Tabla 1. Marco integrado: gestión de ERM 2017 con el COSO}

\begin{tabular}{|l|l|}
\hline \multicolumn{1}{|c|}{ Flujo del proceso } & \multicolumn{1}{c|}{ Componentes del modelo } \\
\hline Misión, visión y valores & Gobierno y cultura \\
\hline Desarrollo de la estrategia & $\begin{array}{l}\text { Estrategias y establecimiento } \\
\text { de objetivos }\end{array}$ \\
\hline $\begin{array}{l}\text { Formulación de objetivos } \\
\text { empresariales }\end{array}$ & Desempeño \\
\hline Implementación y desempeńo & Revisión y monitorización \\
\hline Mejoramiento del valor & $\begin{array}{l}\text { Información, comunicación } \\
\text { y reporte }\end{array}$ \\
\hline
\end{tabular}

\section{Revisión de la literatura e hipótesis}

En el contexto de la actual pandemia, los antecedentes sobre los aspectos de control interno y gestión empresarial conciernen a la limitación de optimizaciones con resistencia al cambio por el capital humano. Estar en la zona de confort es muy atractivo frente a innovaciones, especialmente, de control. Sin embargo, esto impide mejorar resultados, incluso en función a los planes estratégicos institucionales (Gómez-Selemeneva, Blanco, \& Conde, 2013, p.64). 
Con respecto al control interno, se ha mencionado que es multifuncional, pues es aplicable tanto en lo financiero como en lo operativo. Por lo tanto, está presente en todas las funciones de cualquier institución. El control interno tiene el fin de manejar riesgos de todo tipo que impidan objetivos orgánicos o en relación con el mercado (Fernández \& Vásquez, 2014, p. 89). En tal sentido, los registros financieros y operativos deben reflejar la realidad como demostración ética y respaldo de reputación institucional, además del cumplimiento regulatorio de presentación de estados financieros (Serrano, Señalin, Vega \& Herrera, 2017, p. 40).

Para la sociedad en general, es importante alcanzar eficiencia en el control interno con el fin de optimizar la gestión empresarial, especialmente, en el sector comercio, como se ve en esta investigación. El sistema de control interno es inherente a cualquier organización: toda institución lo posee inevitablemente, aunque sea ineficiente. Como las entidades cuentan con bienes y registros, por medio de un adecuado sistema, se busca proteger sus activos y contar con archivos financieros integrales. Si se logran estos dos objetivos mínimos, los resultados serán los mejores a alcanzar (Valqui \& Aguinaga, 2016, p. 49).

El control interno posibilita perfeccionar la gestión operativa al revelar debilidades en el manejo funcional. Esta es una ventaja, pues favorece el uso conveniente de los recursos institucionales para alcanzar sus propios objetivos corporativos. El control, por lo tanto, debe ser cuidadosamente tenido en cuenta por la influencia ejercida en los resultados económicos y financieros, como consecuencia de asegurar razonablemente sus metas en función del desarrollo de una apropiada vigilancia de las obligaciones, responsabilidades y cumplimientos estandarizados (Lamadrid, 2014, p. 76).

Por otro lado, el impacto del control interno en la gestión financiera es importante. Teniendo en cuenta la trascendencia del aspecto inicial de las finanzas (es decir, la contabilidad y su implicancia en el manejo de las cifras de los resultados), la gestión financiera involucra registros, autorizaciones, transacciones, pedidos, endeudamiento, tesorería y cobranzas, con el fin de conseguir el progreso económico o crematístico del negocio. El manejo de la gestión se favorece; especialmente, el correspondiente a las funciones del tesorero o gestor financiero (Burgos \& Suarez, 2017, p. 93).

En este contexto, el impacto de la pandemia en la actividad económica ha sido crítica por las medidas de contención necesarias. Los países han tratado de apoyar financieramente al sector productivo, como es el caso peruano, pero los efectos del COVID-19 han sido superiores a tales esfuerzos. Se promulgaron cuarentena y toques de queda prolongados, los que supusieron el cierre de empresas y pérdidas de empleo. Los sectores más afectados fueron los de hospedaje, restaurantes, transporte aéreo y servicios turísticos, entre otros (PWC, 2020).

Para ahondar en la literatura sobre el tema, a continuación, se presentarán el marco teórico y el marco conceptual. El marco teórico comprende control interno y gestión empresarial. Con respecto al marco conceptual, Paletta y Alimehmeti (2018) afirmaron que las organizaciones con procesos de control interno ineficientes poseen obstáculos superiores de servicios y, por tanto, mayor grado de retribución para niveles altos de la empresa (p. 277). En cambio, para Gupta, Sami y Zhou (2018) el control es relevante en el aspecto financiero, especialmente, cuando la empresa cotiza en bolsa (p. 20). Para Gao y Jia (2017) el control interno influye en la reducción del gasto para obtener recursos patrimoniales; además, los autores señalan que los reportes de deficiencias de control interno tienen consecuencias importantes para los encargados de evaluar riesgos a efectos de la emisión de sus valores (p. 303).

Contabilidad y Negocios (16) 31, 2021 / e-ISSN 2221-724X 
Se debe tener en cuenta que el examen y difusión de los controles internos poseen un impacto importante en las acciones tácticas en las organizaciones (Sun, 2016, p. 277). Así, los fuertes sistemas de controles internos relativos a los reportes financieros son significativos para la emisión de informes financieros válidos y, por lo tanto, confiables (Dickins \& Fay, 2017, p. 117).

Es posible que las empresas que tienen un lineamiento creativo, de apertura a nuevos procesos, sean proclives a tener un control interno con mayor inestabilidad que las firmas que mantienen una línea conservadora o mecanicista en su organización (Bentley-Goode, Newton, \& Thompson, 2017, p. 49). Por ejemplo, cuando comienzan a desarrollarse operativamente, los negocios familiares poseen problemas para poder adecuarse a los cambios y aceleraciones ambientales, de mercado, de legislación y de crecimiento (Başkurt \& Altindağ 2017, p. 10).

La calificación certificada del proceso sistemático de manejo gerencial de responsabilidad social (SR) es beneficiosa para la entidad (Arimany-Serrat \& SabataAliberch, 2018, p. 116). Si se considera activo, el proceso definitorio del ente o de la firma transmite la idea que su accionar asume retos para asumir o entender con fluidez los riesgos de las actividades empresariales, especialmente, en el ámbito gerencial u operativo (Munksgaard \& Ford, 2017, p. 327).

Por otro lado, la actividad intelectual en las empresas (también entendida como el desarrollo científico empresarial) nació paralelamente con la curiosidad de teóricos que han estudiado el manejo gerencial vinculado con la actividad corporativa de los negocios (Carton \& Mouricou, 2017, p. 166). Los indicadores clásicos de evaluación financiera fueron arduamente atacados porque no abarcaban los campos diferentes a lo exclusivamente financiero, como es el caso del grado de creatividad, la investigación, el nivel motivacional, la capacidad innovadora y otros indicadores intelectuales (Hornungová, 2017, p. 71). En este sentido, existe una incidencia importante en lo intencional, en lo creativo y en la decisión personal para adoptar o asumir mayores riesgos entre los alumnos de ciencias empresariales de centros universitarios estatales frente a sus pares de universidades particulares (Raza \& Irfan, 2017, p. 117).

Para ahondar en el marco conceptual, cabe comentar lo establecido desde lo empresarial. A partir de Mantilla (2018), se entiende el "control interno" como el procedimiento en que está inmersa la responsabilidad funcional de todos los integrantes de una entidad, que deben estar involucrados en la optimización de sus funciones y en la búsqueda de la reducción de ineficiencias. Para tal fin, se debe contar con recursos disponibles y suficientes para cumplir los objetivos institucionales. Tales recursos se circunscriben al capital humano y los reportes funcionales.

Con respecto a "gestión empresarial", de acuerdo a Domínguez (2008), se entiende como una actividad que reúne actividades de gestión operativa y de gestión financiera. Por un lado, las de gestión operativa involucran actividades funcionales encaminadas al aprovechamiento de los resultados favorables a partir de la toma de decisiones atinadas y oportunas en beneficio de la organización. Por otro lado, la gestión financiera está enmarcada en las actividades contables con el objeto de emitir y analizar información financiera veraz, integral y con la periodicidad necesaria.

Sobre "responsabilidad funcional", en un estudio elaborado por Medina y Severino (2014), se ha considerado que el concepto representa el grado en que se desenvuelve el grupo de labores que da sentido a la entidad. A partir de ello, en esta investigación, se aplica todo lo programado en los ítems más eleva- 
dos en la organización: incluye departamentos como los de abastecimiento, producción, ventas, y labores de conservación de clientes o marketing (Medina \& Severino, 2014, pp. 65-66).

Por otro lado, con respecto a la optimización de funciones, Chaín-Navarro (1999) desarrolló un estudio a partir de la premisa de que todo servidor labore según su especialidad y, por ello, los resultados son superiores. De esta manera, se logra elevar la productividad funcional (p. 46). En cuanto a la reducción de ineficiencias, de acuerdo con Gallardo (1999), existe una importante cantidad de ejemplos entre lo exigido por una autoridad y una empresa que le presenta su información económica financiera. La entidad supervisora elegirá entre los resultados favorables para la corporación y el nivel de satisfacción de los usuarios del mercado, con el fin de optimizar defectos en la producción o distribución (p. 28).

Con respecto a recursos disponibles, según Chiavenato \& Sapiro (2017), innumerables organizaciones tratan de tener verticalidad con los insumos y la producción para reducir su vulnerabilidad frente a los abastecedores; al mismo tiempo, buscan poder controlar sus gastos productivos para disponer o liberar mayores recursos, y tienen la posibilidad de la tercerización con el fin de especializarse en la parte medular de sus operaciones (p. 28). En relación a capital humano, Torres (2011) ha señalado que los recursos humanos son el principal componente de la gestión. Por ello, se debe impulsar su adecuado manejo gerencial para mantenerlo motivado (p. 152). Sobre reportes funcionales, De Souza, Suzacq y Torrendell (2009) han manifestado que, en grandes instituciones, lo más resaltante organizativamente es la responsabilidad y asunción de funciones. Las características de cada agencia, sucursal o subsidiaria no son fácilmente percibidas con precisión, por lo que las decisiones se pueden postergar innecesariamente (p.61).
Con relación a la gestión operativa, Serra (2005) ha indicado que los gerentes con múltiples campos geográficos tienen el reto de actuar teniendo presentes distintas realidades o momentos. Así, debe saber compaginar los objetivos de la matriz con los pensamientos y actuaciones locales (p. 9). Con respecto a las actividades funcionales, según Estevez, Jones y Anderson (2002), estas están sujetas a ser un conglomerado, pero en función al manejo gerencial, del cual dependen (p. 9).

Acerca de la toma de decisiones, según Solano (2003), los gestores o gerentes están en constante actuación empresarial; así, sus preocupaciones no son solo operativas internas, sino también de repercusión en el mercado, de acuerdo a los resultados de su gestión (p. 44). En lo que respecta a la gestión financiera, según Nava (2009), esta tiene el propósito de maximizar el valor de la organización; es decir, si el caso lo amerita, la cotización de las acciones debe estar en la agenda del administrador financiero.

En un trabajo sobre "actividades contables", González (2006) ha definido con precisión dicho término como las labores dirigidas a favorecer la función de control y registrar activos, gastos, balances, resultados, etc. (p. 35). Sobre la información financiera, Nava (2009) ha manifestado que, en la época moderna, incorpora labores tendentes a optimizar los resultados empresariales. Para ello, se vale de recursos digitales o impresos, con reportes de los estados financieros en su etapa final o como informe operativo de uso gerencial.

Además de los conceptos presentados, cabe indicar precisiones sobre el contexto correspondiente a la investigación. La pandemia del COVID-19 afectó el comercio textil del emporio de Gamarra; y el mercado de comestibles más importante, que es el sector de La Parada. Como consecuencia, hubo desabastecimiento temporal alimenticio y leve alza inflacionaria 
con caída del PBI, del 11,6\% para el año 2020 en Perú. Asimismo, los efectos de la pandemia repercutieron en inestabilidad económica y descontento popular en los negocios comerciales (Food and Agriculture Organization of the United Nations [FAO], 2020).

Frente a la información presentada, este trabajo ha partido del siguiente problema de investigación: ¡en qué nivel se relaciona el control interno con la gestión empresarial de centros comerciales en tiempos de COVID-19? Como hipótesis, se planteó que el control interno se relaciona significativamente con la gestión empresarial de centros comerciales en tiempos de COVID-19. El objetivo de la investigación ha consistido en analizar en qué nivel se relaciona el control interno con la gestión empresarial de centros comerciales en tiempos de COVID-19.

Con respecto a la justificación, cabe mencionar que el control interno es una instrumentalización prioritaria de uso gerencial para relacionarla, directamente, con la gestión empresarial. Así, es factible optimizar procesos de control que la auditoría pertinente evaluará. De esta manera, se coadyuva a los esfuerzos gerenciales en obtener resultados satisfactorios con manejos sanos, oportunos y beneficiosos en los comerciantes del distrito La Victoria en Lima. Sin embargo, la pandemia del COVID-19 alteró a todo el planeta en todas las actividades. Antes de esta realidad, en Perú, la informalidad laboral correspondía al 70\% de las empresas: Para enero 2021, bordeaba el 80\%. Asimismo, en ese lapso de tiempo, se perdieron más de dos millones de empleos (Espinoza \& Arias, 2020).

\section{Metodología}

De acuerdo con la hipótesis del trabajo, en la tabla 2 , se muestra el cuadro de operacionalización de las variables control interno y gestión empresarial, y la definición conceptual de ellas, así como de sus dimensiones e indicadores.

Tabla 2. Cuadiro de operacionalización de variables

\begin{tabular}{|c|c|c|c|c|}
\hline Hipótesis & Variable & Definición conceptual & Dimensiones & Indicadores \\
\hline \multirow{8}{*}{$\begin{array}{l}\text { En tiempos de } \\
\text { COVID-19, el } \\
\text { control interno se } \\
\text { relaciona significati- } \\
\text { vamente a la gestión } \\
\text { empresarial de cen- } \\
\text { tros comerciales. }\end{array}$} & \multirow[t]{4}{*}{$\begin{array}{l}\text { Control } \\
\text { interno }\end{array}$} & \multirow{4}{*}{$\begin{array}{l}\text { Se concluye que el control interno es un proceso en que } \\
\text { está inmersa la responsabilidad funcional de todos los } \\
\text { integrantes de una entidad, quienes deben estar invo- } \\
\text { lucrados en la optimización de sus funciones y en la } \\
\text { búsqueda de la reducción de ineficiencias. Para tal fin, } \\
\text { se debe contar con recursos disponibles suficientes para } \\
\text { cumplir los objetivos institucionales. Tales recursos se cir- } \\
\text { cunscriben al capital humano y los reportes funcionales }\end{array}$} & \multirow[t]{2}{*}{$\begin{array}{l}\text { Responsabili- } \\
\text { dad funcional }\end{array}$} & $\begin{array}{l}\text { 1. Optimización de } \\
\text { funciones }\end{array}$ \\
\hline & & & & $\begin{array}{l}\text { 2. Reducción de inefi- } \\
\text { ciencias }\end{array}$ \\
\hline & & & \multirow{2}{*}{$\begin{array}{l}\text { Recursos } \\
\text { disponibles }\end{array}$} & 3. Capital humano \\
\hline & & & & 4. Reportes funcionales \\
\hline & \multirow[t]{4}{*}{$\begin{array}{l}\text { Gestión } \\
\text { empresarial }\end{array}$} & \multirow{4}{*}{$\begin{array}{l}\text { Se entiende la gestión empresarial como una actividad } \\
\text { que reúne actividades de gestión operativa y gestión } \\
\text { financiera. Por un lado, la gestión operativa involucra } \\
\text { actividades funcionales encaminadas al aprovechamiento } \\
\text { de los resultados favorables en la toma de decisiones ati- } \\
\text { nadas y oportunas en beneficio de la organización. Por } \\
\text { otro lado, la gestión financiera está enmarcada en las } \\
\text { actividades contables con el objeto de emitir y analizar } \\
\text { información financiera veraz, integral y con la periodi- } \\
\text { cidad necesaria. }\end{array}$} & \multirow[t]{2}{*}{$\begin{array}{l}\text { Gestión } \\
\text { operativa }\end{array}$} & $\begin{array}{l}\text { 5. Actividades funcio- } \\
\text { nales }\end{array}$ \\
\hline & & & & 6. Toma de decisiones \\
\hline & & & \multirow[t]{2}{*}{$\begin{array}{l}\text { Gestión } \\
\text { financiera }\end{array}$} & $\begin{array}{l}\text { 7. Actividades con- } \\
\text { tables }\end{array}$ \\
\hline & & & & $\begin{array}{l}\text { 8. Información finan- } \\
\text { ciera }\end{array}$ \\
\hline
\end{tabular}

Fuente: Elaborado sobre la base de la tematización sobre control interno (Mantilla, 2018) y gestión empresarial (Domínguez, 2008). 
El tipo de investigación planteada es descriptivo-correlacional al contar con materias, tópicos, temas, dimensiones, categorías, y clases en un ámbito específico. Su diseño es no experimental y transversal (Hernández, Fernández, \& Baptista, 2014, pp. 54, 93, 152). Para conformar la población, han sido considerados los cinco centros comerciales más importantes de La Victoria en Lima. De ellos, se eligieron a 45 responsables de las áreas contable, productiva y tesorería. Entre los participantes, hubo propietarios, gerentes o administradores. Se aplicó el muestreo con censo para que, con los criterios de selección, la muestra fuese la misma, pues, así, se incluyen todos los elementos (Hernández et al., 2014, p. 172). La técnica utilizada fue la encuesta. Se tomó como instrumento el cuestionario con trabajo de campo, que fue sujeto a validación (Hernández et al., 2014, p. 200), con fiabilidad medida a través del alfa de Cronbach. El procedimiento de recolección de datos se efectuó aplicando los cuestionarios para ambas variables. Posteriormente, se trasladaron los datos a una hoja de cálculo de Excel y luego se procesaron por medio de SPSS. El método de análisis de datos utilizado fue el deductivo (Ayala, 2021). En cuanto a los aspectos éticos, se consideró citar a los autores de las fuentes originales.

\section{Resultados}

Para obtener los resultados, se validó y se calculó la fiabilidad del instrumento utilizado en la investigación, con el fin de probar la normalidad y la correlación entre variables:

\section{Tabla 3. Validación V Aiken}

\begin{tabular}{|l|c|}
\hline \multicolumn{1}{|c|}{ Características evaluadas } & V Aiken \\
\hline Relevancia & $100 \%$ \\
\hline Pertinencia & $100 \%$ \\
\hline Claridad & $100 \%$ \\
\hline Promedio & $100 \%$ \\
\hline
\end{tabular}

Fuente: Elaboración sobre la base de los juicios de los autores revisados
La tabla 3 muestra la validez del instrumento empleado para las variables, las dimensiones y los indicadores. Su valoración ha sido plenamente favorable (Hernández et al., 2014).

\section{Tabla 4. Estadísticas de fiabilidad}

\begin{tabular}{|c|c|}
\hline Alfa de Cronbach & $\mathrm{N}^{\circ}$ de elementos \\
\hline, 885 & 20 \\
\hline
\end{tabular}

Fuente: Elaboración propia a partir de la base de datos.

La tabla 4 muestra la fiabilidad del instrumento empleado, cuyo cálculo corresponde al nivel "muy bueno", pues se ubica en el rango que va desde mayor a 0,7 hasta 0,9 (Tuapante, Duque, \& Mena, 2017, p. 41).

Tabla 5. Prueba de normalidad de las variables

\begin{tabular}{|l|c|c|c|}
\hline & $\begin{array}{c}\text { Estadístico } \\
\text { Shapiro-Wilk }\end{array}$ & gl & Sig. \\
\hline Control interno &, 951 & 45 &, 056 \\
\hline Gestión empresarial &, 935 & 45 &, 015 \\
\hline
\end{tabular}

Fuente: Elaboración propia a partir de la base de datos.

La tabla 5 muestra la prueba de normalidad para las variables con el estadístico Shapiro-Wilk por tener un gl $\leq 50$, y una significación $(\alpha=5 \%)$ e hipótesis estadística, nula y alterna:

- H0: Los datos no difieren de una distribución normal.

- H1: Los datos difieren de una distribución normal.

Se decidió utilizar el Rho de Spearman para probar en qué nivel el control interno se relaciona con la gestión empresarial de centros comerciales en tiempos de COVID-19, debido a que los dos p valor no cumplen el requisito de ser ambos $\leq 0,05$, lo que implica aceptar la $\mathrm{H} 1$ y aplicar prueba no paramétrica.

Contabilidad y Negocios (16) 31, 2021 / e-ISSN 2221-724X 
Tabla 6. Prueba de correlación entre las variables

\begin{tabular}{|l|c|c|c|}
\hline \multicolumn{4}{|c|}{ Gestión empresarial } \\
\hline \multirow{2}{*}{ Control interno } & Rho de Spearman & p valor & N \\
\cline { 2 - 4 } &, 778 &, 000 & 45 \\
\hline
\end{tabular}

Fuente: Elaboración propia a partir de la base de datos.

La tabla 6 muestra la prueba de correlación entre las variables, con una significación $(\alpha=5 \%)$ e hipótesis nula y alterna:

- H0: El control interno no se relaciona significativamente con la gestión empresarial de centros comerciales en tiempos de COVID-19.

- H1: El control interno se relaciona significativamente con la gestión empresarial de centros comerciales en tiempos de COVID-19.

Se observa el $p$ valor $\leq 0,05$, por lo que se acepta la $\mathrm{H} 1$. Además, el estadístico Rho de Spearman es positivo, directo y un valor alto. En consecuencia, el control interno se relaciona significativamente con la gestión empresarial de centros comerciales en tiempos de COVID-19. Por lo tanto, si sube la primera variable, también lo hará la segunda y viceversa.

Tabla 7. Prueba de correlación entre dimensiones

\begin{tabular}{|l|c|c|c|}
\hline \multirow{4}{|c|}{ Gestión operativa } \\
\hline \multirow{2}{*}{ Control interno } & Rho de Spearman & p valor & N \\
\cline { 2 - 4 } &, 718 &, 000 & 45 \\
\hline
\end{tabular}

Fuente: Elaboración propia a partir de la base de datos.

La tabla 7 muestra la prueba de correlación entre las variables, con una significación $(\alpha=5 \%)$ e hipótesis nula y alterna:

- H0: El control interno no se relaciona significativamente con la gestión operativa de centros comerciales en tiempos de COVID-19.
- H1: El control interno se relaciona significativamente con la gestión operativa de centros comerciales en tiempos de COVID-19.

Como se observa, el $\mathrm{p}$ valor es $\leq 0,05$, por lo que se acepta la H1. Además, el estadístico Rho de Spearman es positivo, directo y un valor alto. En consecuencia, el control interno se relaciona significativamente con la gestión operativa de centros comerciales en tiempos de COVID-19. Por tanto, si sube la primera variable, también lo hará la segunda y viceversa.

Tabla 8. Pruebas de correlación entre dimensiones

\begin{tabular}{|l|c|c|c|}
\hline \multicolumn{4}{|c|}{ Gestión financiera } \\
\hline \multirow{2}{*}{ Control interno } & Rho de Spearman & p valor & N \\
\cline { 2 - 4 } &, 705 &, 000 & 45 \\
\hline
\end{tabular}

La tabla 8 muestra la prueba de correlación entre las variables, con una significación $(\alpha=5 \%)$ e hipótesis nula y alterna:

- H0: El control interno no se relaciona significativamente con la gestión financiera de centros comerciales en tiempos de COVID-19.

- H1: El control interno se relaciona significativamente con la gestión financiera de centros comerciales en tiempos de COVID-19.

A partir de lo presentado, el control interno se relaciona significativamente con la gestión empresarial en tiempos de COVID-19, no solo en Perú, sino también internacionalmente. Esto se debe a que se ha reducido personal; además, han aumentado las actividades virtuales y remotas, lo que ha supuesto el uso de mayor modernidad tecnológica (Quevedo, Vásquez, Quevedo, $\&$ Pinzon, 2020). Igualmente, se comprobó que existe relación significativa en tiempos de COVID-19 entre el control interno con las gestiones operativa y financiera. 


\section{Discusión}

En cuanto a la relación entre las variables de control interno y gestión empresarial, en el trabajo de investigación de Valqui y Aguinaga (2016), se resalta la importancia para la sociedad en su conjunto de alcanzar eficiencia en el control interno para mejorar la gestión empresarial. Toda organización cuenta con control: puede tener un sistema ineficiente, pero es imposible su inexistencia, pues hay operaciones, registros y normas. Las instituciones tienen bienes y registros. Un apropiado sistema trata de salvaguardar sus activos, por lo que cuenta con archivos financieros lo más fieles posibles para dicho objetivo. Al lograr estos dos propósitos básicos, los resultados serán los óptimos a conseguir. En el presente artículo, se comprobó, a través del análisis estadístico, la relación significativa entre las dos variables en tiempos de COVID-19. Además, se verificó que la relación estudiada es directa. Por lo tanto, si hay mejor control interno, habrá mejor gestión empresarial y viceversa. Es decir, comparativamente, existe coincidencia. Para tal fin, se debe entender que las acciones ejecutadas en una empresa en relación a las dos variables están interrelacionadas por el manejo gerencial. En otras palabras, la tendencia del control interno en los centros comerciales mencionados se refleja en el mismo sentido en la gestión empresarial de esas entidades.

Adicionalmente, la pandemia ha producido que las empresas implanten modificaciones internas en el manejo empresarial. Frente a ello, los servidores luchan con el estrés que redundan en la comunicación con incertidumbre. En el caso de Perú, el cierre de los centros comerciales en dos cuarentenas entre 2020 y 2021 afectó a los negocios, pues la mayoría son conglomerados, como los que abundan en La Victoria; y al bienestar. Es decir, los negocios con adecuado control interno y gestión empresarial fueron tratados de la misma manera que aquellos que no tienen esas cualidades (Li, Sun, Tao, \& Lee, 2021).

Con respecto a la relación entre control interno y gestión operativa, en la investigación desarrollada por Lamadrid (2014), se llega a mencionar que el control interno posibilita perfeccionar la gestión operativa al revelar debilidades en el manejo funcional. Esta es una ventaja, pues favorece el uso conveniente de los recursos institucionales y permite alcanzar sus propios objetivos corporativos. Por lo tanto, el control debe ser cuidadosamente tenido en cuenta por la influencia ejercida en los resultados económicos y financieros, como consecuencia de asegurar razonablemente sus metas en función del desarrollo de una apropiada vigilancia de las obligaciones, responsabilidades y cumplimientos estandarizados. En la presente investigación, se comprobó que el control interno se relaciona significativamente con la gestión operativa en los centros comerciales en tiempos de COVID-19. Asimismo, se evidenció que la relación verificada es directa; es decir, si hay mejor control interno, habrá mejor gestión operativa y viceversa. Entonces, se encuentran coincidencias con la investigación de Lamadrid. Por ello, cuando existen debilidades en el sistema de control interno, estas se reflejan en los resultados de la gestión operativa. Una característica intrínseca del control consiste en buscar, paralelamente, tanto el uso eficiente de los recursos de las entidades comerciales como el aseguramiento razonable de alcanzar las metas propuestas en el ámbito operativo.

Asimismo, se sabe que las instituciones actúan en un ámbito generalizado de control estructural y de funciones operativas interconectadas. En tal sentido, los comerciantes están expuestos a los cambios mundiales, como es el caso de las consecuencias del COVID-19. En Perú, a febrero 2021, el virus infectó a más de un 
millón de personas, y causó la muerte de más de cuarenta mil; además, supuso recesión extrema, especialmente, en el sector de comercio (Komljenovic, 2021).

Respecto de la relación entre control interno y gestión financiera, en su trabajo investigativo, Burgos y Suarez (2017) han manifestado que la correspondencia del control interno es sustancial sobre la gestión financiera. Cuando los sistemas transaccionales, funcionales y de responsabilidad se alinean con parámetros o estándares preestablecidos, tienen comportamiento positivo en los objetivos trazados en el campo financiero, especialmente, en el manejo de la tesorería, con el fin de contribuir con el desarrollo de los negocios. En el presente artículo, se demostró que el control interno se relaciona significativamente con la gestión financiera en los centros comerciales en tiempos de COVID19. Finalmente, se verificó que la relación investigada es directa, pues, si hay mejor control interno, habrá mejor gestión financiera y viceversa. Por lo visto anteriormente, los dos trabajos coinciden en los aspectos expresados. Por tanto, el control interno y la gestión financiera están enraizados uno en el otro cuando los registros, funciones y obligaciones se establecen con los propósitos perseguidos. De esa manera, la parte financiera y la de tesorería se benefician cuando tales actividades funcionales son cumplidas con eficiencia, efectividad y economicidad.

En ese sentido, si el control falla, el riesgo financiero perjudica a las compañías. En la pandemia, el comportamiento del personal debe ajustarse a protocolos con los clientes y conductas obligatorias, como el uso de mascarillas, el lavado de manos y el distanciamiento social. Así, el incumplimiento regulatorio de medidas de emergencia sanitaria ha llevado a multas pecuniarias, pero no ha disminuido la inseguridad ciudadana, con incidencia aún mayor en los negocios (Frank \& Greenman, 2020).

\section{Conclusiones}

La investigación partió del objetivo de analizar en qué nivel el control interno (Mantilla, 2018) se relaciona con la gestión empresarial (Domínguez, 2008) en los centros comerciales en tiempos de COVID-19. Para ello, se utilizaron instrumentos validados (Hernández et al., 2014) y debidamente fiables (Tuapante et al., 2017). A partir de ello, se llegó a las conclusiones que se comentarán a continuación

En primer lugar, el control interno se relaciona significativamente (según la encuesta, en $77,8 \%$ de los negocios) con la gestión empresarial en los centros comerciales en tiempos de COVID-19; además, se observa que la relación estudiada es directa. Entonces, si hay mejor control interno, habrá mejor gestión empresarial y viceversa. Para este caso, se observó que la relación entre la responsabilidad funcional y los recursos disponibles es simultáneamente fuerte con la gestión operativa y financiera.

En segundo lugar, el control interno se relaciona significativamente (según la encuesta, en 71,8\% de los negocios) con la gestión operativa en los centros comerciales en tiempos de COVID-19; también se advierte que la relación estudiada es directa. Para este caso, se observó que la relación entre la responsabilidad funcional y recursos disponibles es concurrentemente fuerte con las actividades funcionales y la toma de decisiones.

En tercer lugar, el control interno se relaciona significativamente (según la encuesta en, 70,5\% de los negocios) con la gestión financiera en los centros comerciales en tiempos de COVID-19. Además, se contempla que la relación estudiada es directa. Para este caso, se ha observó que la relación entre la responsabilidad funcional y los recursos disponibles es 
coincidentemente fuerte con las actividades contables y la información financiera. Se debe aclarar que la referencia al COVID-19 indica solo el periodo bajo estudio (2020) y no la medición del impacto respecto de periodos anteriores.

En concordancia con lo concluido, se generaron las siguientes recomendaciones en relación con el contexto del COVID-19. En primer lugar, la responsabilidad funcional en los centros comerciales debe ser impulsada por los encargados de las áreas de contabilidad, producción y tesorería, así como por los gerentes o administradores. Para ello, la inversión en recursos disponibles debe ser correspondiente directamente con el manejo operativo y financiero a establecer, dado el incremento de actividades virtuales por razones de salud. Para efectos de futuras investigaciones, se sugiere ampliar la investigación científica a una muestra o población con mayor tamaño de ejecutores y propietarios de negocios, así como a la inmensa diversidad de otros sectores económicos, como el industrial, el de servicios, el minero, el pesquero, etc.

En segundo lugar, la optimización de funciones en los centros comerciales debe ser aplicada con reducción de ineficiencias. Para ello, el control interno debe estar en concordancia continua con las actividades funcionales y la toma de decisiones, dada la reducción de labores presenciales y el incremento de trabajo remoto. Para efectos de próximas investigaciones, se propone ampliar la investigación científica a un ámbito geográfico mayor al empleado en este estudio; es decir, se plantea que comprenda no solo un distrito, sino el país y el extranjero, comenzando por casos de Sudamérica por las economías similares imperantes en la mayoría de las naciones en esta parte del continente.

En tercer lugar, el capital humano en los centros comerciales debe estar orientado con reportes fun- cionales. Para ello, el control interno debe estar en concomitancia evidente con las actividades contables e información financiera dadas las nuevas modalidades de servicio por internet, delivery, con contactos bancarios y financieros electrónicos. Para efectos de posteriores investigaciones, se plantea ampliar este artículo a mayor número de períodos de tiempo a los dedicados para este estudio. Asimismo, se propone que se incluyan otras dimensiones o variables con sus respectivos indicadores, acordes con los nuevos enfoques a darse circunstancialmente, según sea requerido para la contribución del conocimiento en estas materias, especialmente, en épocas de alto riesgo como la actual crisis sanitaria mundial e, inclusive, en tiempos de postpandemia.

\section{Referencias bibliográficas}

Arimany-Serrat, N., \& Sabata-Aliberch, A. (2018). Social Responsibility as a Management System. Intangible Capital, 14(1), 116-126. https://doi.org/10.3926/ic.1105

Başkurt, G., \& Altindağ, E. (2017). The Impact of Institutionalization of Family Business on Strategic Human Resources Management and Company Performance. Business Management Dynamics, 7(3), 10-25.

Bentley-Goode, K.A., Newton, N.J., \& Thompson, A.M. (2017). Business Strategy, Internal Control over Financial Reporting, and Audit Reporting Quality. Auditing: A Journal of Practice \& Theory, 36(4), 49-69. https://doi.org/10.2308/ajpt-51693

Burgos, C.E., \& Suarez, R.H. (2017). El sistema de control interno contable y su influencia en la gestión financiera de la empresa inversiones Christh.Al. SAC, año 2015. (Tesis de licenciatura, Facultad de Ciencias Económicas, Universidad Privada Antenor Orrego. Lima, Perú).

Ayala, M. (2021, 22 de febrero). ¿Qué son los métodos de investigación? Lifeder. Recuperado de https://bit. ly/2MXOMNM [Consulta: 30 de abril de 2021].

Contabilidad y Negocios (16) 31, 2021 / e-ISSN 2221-724X 
Carton, G., \& Mouricou, P. (2017). Is Management Research Relevant? A Systematic Analysis of the Rigor-Relevance Debate in Top-Tier Journals (19942013).M@N@Gement, 20(3),166-203. https://doi. org/10.3917/mana.202.0166

Chaín-Navarro, C. (1999). Gestión de información para la investigación: Desarrollo y consolidación de las funciones del gestor como asesor y consultor para la ciencia y la tecnología. Ciencias de la Información, 30(4), 39-50.

Chiavenato, I., \& Sapiro, A. (2017). Planeación estratégica. México, D. F.: McGraw-Hill Interamericana.

De Souza, M., Suzacq, A., \& Torrendell, S. (2009). Rol y responsabilidad del contador público y del licenciado en Administración en la generación de información y toma de decisiones. (Trabajo Final de Grado, Facultad de Ciencias Económicas y de Administración, Universidad de la República. Montevideo, Uruguay).

Dickins, D., \& Fay, R.G. (2017). COSO 2013: Aligning Internal Controls and Principles. Issues in Accounting Education, 32(3), 117-127. https://doi.org/10.2308/ iace-51585

Domínguez, P.R. (2008). Introducción a la gestión empresarial. Madrid: B-EUMED, Instituto Europeo de Gestión Empresarial.

Espinoza, C., \& Arias, J. (2020). COVID-19 Effect on Herding Behaviour in European Capital Markets. Finance Research Letters, 38. https://doi.org/10.1016/j. frl.2020.101787

Estevez, A.M., Jones, C.O., \& Anderson, J. (2002). El modelo secuencial de politicas públicas treinta años más tarde. Buenos Aires: Centro de Investigación en Administración Pública, Universidad de Buenos Aires

Fernández, S., \& Vásquez, F. (2014). El control interno y su influencia en la gestión de las empresas privadas de Latinoamérica, Perú. Crescendo Ciencias Contables \& Administrativas, 1(1), 89-98. https://doi. org/10.21895/in cres ccff.v1i1.247
Food and Agriculture Organization of the United Nations (FAO). (2020). Análisis del mercado del banano: Panorama general de febrero de 2020. Recuperado de fao.org/3/ ca9212es/ca9212es.pdf [Consulta: 30 de abril de 2021].

Frank, J., \& Greenman, L. (2020). Revisiting Conduct Risk Management in the COVID-19 Era with Updated DOJ Criteria. Journal of Risk Management in Financial Institutions 13(4), 295-307.

Gallardo, J. (1999). Disyuntivas en la teoría normativa de la regulación: El caso de los monopolios naturales (Documento de trabajo 164). Lima: Pontificia Universidad Católica del Perú. Recuperado de http://files.pucp. edu.pe/departamento/economia/DDD164.pdf [Consulta: 30 de abril de 2021].

Gao, X., \& Jia, Y. (2017). The Role of Internal Control in the Equity Issue Market: Evidence from Seasoned Equity Offerings. Journal of Accounting, Auditing \& Finance, 32(3), 303-328. https:/doi. org/10.1177/0148558X15602821

Gómez, G. (2001, mayo 11). Control interno en la organización empresarial. Gestiopolis. Recuperado de https:// bit.ly/2uiVrai [Consulta: 30 de abril de 2021].

Gómez-Selemeneva, D., Blanco, B., \& Conde, J.R. (2013). Internal Control System for the Improvement of Corporate Governance in Cuba. GECONTEC: Revista Internacional de Gestión del Conocimiento y la Tecnología, 1(2), 53-65. Recuperado de https://bit. ly/2FO7u5R [Consulta: 30 de abril de 2021].

González, J.A.R. (2006). De la estructura por funciones al enfoque basado en procesos y a la visión sistémica de la organización. Revista Ciencias Estratégicas, 14(15), $33-42$.

Gupta, P.P., Sami, H., \& Zhou, H. (2018). Do Companies with Effective Internal Controls Over Financial Reporting Benefit from Sarbanes-Oxley Sections 302 and 404? Journal of Accounting, Auditing \& Finance, 33(2), 200-227. https://doi.org/10.1177/ $0148558 \times 16663091$ 
Hernández, R., Fernández, C., \& Baptista, P. (2014). Metodología de la investigación cientifica (6a ed.). México, D. F.: Mc Graw Hill Education.

Hornungová, J. (2017). Nonfinancial Performance Evaluation as Significant Area of Strategic Business Management. Business: Theory \& Practice, 18(1), 71-78. https://doi.org/10.3846/btp.2017.008

Komljenovic, D. (2021) Engineering Asset Management at Times of Major, Large-Scale Instabilities and Disruptions. En A. Crespo et al. (eds.), Lecture Notes in Mechanical Engineering (pp. 255-270). Nueva York: Springer. https://doi.org/10.1007/978-3-030-64228$0 \_22$

Lamadrid, P. (2014). Propuesta de diseño de un sistema de control interno para mejorar la gestión operativa de la entidad Yallegué.com dedicada al comercio electrónico de productos diversos en el periodo 2013-2014. (Tesis de licenciatura, Facultad de Ciencias Empresariales, Universidad Católica Santo Toribio de Mogrovejo. Chiclayo, Perú).

Li, J.-Y., Sun, R., Tao, W., \& Lee, Y. (2021). Employee coping with organizational change in the face of a pandemic: The role of transparent internal communication. Public Relations Review, 47(1), 101984. https://doi.org/10.1016/j.pubrev.2020.101984

Mantilla, S. (2018). Auditoría del control interno (4a ed.). Bogotá: ECOE Ediciones.

Medina, A., \& Severino, P. (2014). Responsabilidad empresarial: generación de capital social de las empresas. Contabilidad y Negocios, 9(17), 63-72.

Munksgaard, K.B., \& Ford, D. (2017). The business actor and business management. IMP Journal, 11(2), 327347. https://doi.org/10.1108/IMP-06-2015-0022

Nava, M. (2009). Análisis financiero: Una herramienta clave para una gestión financiera eficiente. Revista Venezolana de Gerencia, 14(48), 606-628. Recuperado de https:// bit.ly/2T0tbTU [Consulta: 30 de abril de 2021]. https://doi.org/10.31876/revista.v14i48.10553
Navia-Núńez, M., \& Stefanu-Lidorikiotu, Y. (2020). Cambios esperados en las funciones administrativas después de la emergencia sanitaria: estudio en directivos de España y Colombia. Contabilidad y Negocios, 15(30), 6-23. https://doi.org/10.18800/contabilidad.202101.001

Paletta, A., \&Alimehmeti, G. (2018). SOXDisclosure and the Effect of Internal Controls on Executive Compensation. Journal of Accounting, Auditing \& Finance, 33(2), 277295. https://doi.org/10.1177/0148558X16630445

PriceWaterhouseCoopers (PWC). (2020). A fondo: Implicaciones contables de los efectos del coronavirus. Recuperado de https://pwc.to/3e4aGuO [Consulta: 30 de abril de 2021].

Quevedo, M., Vásquez, L., Quevedo, J., \& Pinzon, L. (2020). COVID-19 y sus efectos en el comercio internacional. Caso Ecuador. Dominio de las Ciencias, 6(3), 1006-1015. https://doi.org/10.23857/dc.v6i3.1330

Raza, S.A., \& Irfan, M. (2017). Investigating the Intentions, Self-Efficacy and Motivators behind Entrepreneurial Decisions of Business Students. Bulletin of Education \& Research, 39(3), 117-129.

Redacción El Popular (2020, 31 de enero). PNP decomisa polos y buzos de la selección "bambas". El Popular. Recuperado de https://bit.ly/38O7zo3 [Consulta: 30 de abril de 2021].

Serra, A. (2005). La gestión transversal. Expectativas y resultados. Revista del CLAD Reforma y Democracia, (32), 1-17.

Serrano, P.A., Señalin, L.O., Vega, F.Y., \& Herrera, J.N. (2017). El control interno como herramienta indispensable para una gestión financiera y contable eficiente en las empresas bananeras del cantón Machala (Ecuador). Revista Espacios, 39(3), 30-42.

Solano, A.I. (2003). Toma de decisiones gerenciales. Tecnología en Marcha, 16(3), 44-51.

Sun, Y. (2016). Internal Control Weakness Disclosure and Firm Investment. Journal of Accounting, Auditing \& Finance, 31(2), 277-307. https://doi.org/10.1177/ $0148558 X 15598027$

Contabilidad y Negocios (16) 31, 2021 / e-ISSN 2221-724X 
Torres, J.L. (2011). Enfoques para la medición del impacto de la gestión del capital humano en los resultados de negocio. Revista Científica Pensamiento y Gestión, (18), 151-176.

Tuapante, J.V., Duque, M.A., \& Mena, A.P. (2017). Alfa de Cronbach para validar cuestionario de uso de TIC. mktDescubre, 1(10), 37-48. https://doi.org/10.36779/ mktdescubre.v10.141

Valqui, G.L., \& Aguinaga, J.J.T. (2016). El sistema de control interno: Una herramienta para el perfeccionamiento de la gestión empresarial en el sector construcción. Revista de Investigación de Contabilidad Accounting Power for Business, 1(1), 49-59.
Yong, N. (2018, 8 de enero). El valor de una visión conjunta de gestión de riesgos y control interno. Gestión. Recuperado de https://bit.ly/39Ih2ga [Consulta: 30 de abril de 2021].

Fecha de recepción: 04 de febrero de 2021

Fecha de aceptación: 16 de abril de 2021

Correspondencia: espinozauniversidad@gmail.com

erickauni2018@gmail.com tecnologias002016@gmail.com 